\title{
Design, Synthesis and Characterization of Novel Amine Derivatives of 5-[5-(Chloromethyl)-1, 3, 4-Oxadiazol-2-yl]- 2-(4-Fluorophenyl)-Pyridine as a New Class of Anticancer Agents
}

\author{
Adimule Vinayak ${ }^{1,3}$, Medapa Sudha ${ }^{2}$ and Kumar S. Lalita ${ }^{3}$ \\ ${ }^{1}$ Mount Carmel Centre for Scientific Research and Advanced Learning, Mount Carmel College, Vasanthnagar, \\ Bengaluru-560 052, Karnataka, India \\ ${ }^{2}$ Department of Chemistry, Mount Carmel College (Autonomous), Vasanthnagar, Bengaluru-560 052, \\ Karnataka, India \\ ${ }^{3}$ Chemistry Discipline, School of Sciences, IGNOU, New-Delhi, India
}

(Received: January 07, 2016; Accepted: January 16, 2017; Published (web): June 21, 2017)

\begin{abstract}
A linear strategy was adopted in synthesizing the novel amine derivatives 7(a-h) of 5-[5(chloromethyl)-1, 3, 4-oxadiazol-2-yl]-2-(4-fluorophenyl)-pyridine (6) and screened these compounds for in vitro anticancer activity against three human cancer cell lines (HeLa,Caco-2 and HepG2). The synthesised novel compounds were characterized by ${ }^{1} \mathrm{H}$ NMR, MS and ${ }^{13} \mathrm{C}$ NMR spectroscopic evidences. Microwave irradiation of compound (5) in presence of chloroacetyl chloride and phosphoryl oxychloride yielded the dehydrated cyclized key intermediate 5-[5-(chloromethyl)-1,3,4-oxadiazol-2-yl]-2-(4-fluorophenyl)-pyridine which upon treatment with various primary or secondary amines (a-h) resulted into the corresponding amine derivatives. $\mathrm{The}^{\mathrm{I}} \mathrm{IC}_{50}$ values of the final compounds were compared with that of 5-fluorouracil (5-FU) taken as the standard. Compounds 7a and 7d were found to be highly cytotoxic against $H e p G 2$ cell lines with $\mathrm{IC}_{50}$ values of $2.6 \mu \mathrm{M}\left(\mathrm{IC}_{50}=34.0 \pm 0.5 \mu \mathrm{M}\right)$ and $5.8 \mu \mathrm{M}$ $\left(\mathrm{IC}_{50}=112 \pm 1.4 \mu \mathrm{M}\right)$ respectively. The compound (7f) alone was found to have high cytotoxicity against Caco-2 cell lines with $\mathrm{IC}_{50}$ value of $2.3 \mu \mathrm{M}\left(\mathrm{IC}_{50}=87 \pm 2.6 \mu \mathrm{M}\right)$.
\end{abstract}

Key words: Anticancer, $\mathrm{HeLa}, 1,3$, 4-Oxadiazoles, $\mathrm{Hep} G 2$, cytotoxicity

\section{INTRODUCTION}

As per literature review, various analogues of 2, 5-disubstituted-1, 3,4-oxadiazole derivatives of pyridine (Figure 1,C) have been studied for their analgesic, anti-inflammatory ${ }^{1}$, antimicrobial ${ }^{2,3}$, antitumor $^{4}$ and anticancer ${ }^{5}$ properties. Substitution of $4-$ fluorophenyl group at second position of the pyridine containing 1,3,4-oxadiazolesmoiety ${ }^{6}$ has shown very good cytotoxicity against human cancer cell lines ${ }^{7}$. In view of this, novel amine derivatives of 5-[5(chloromethyl)-1,3,4-oxadiazol-2-yl]-2-(4-fluorophenyl)-pyridine (Figure 1, A) have been designed and synthesised. These novel 1,3,4-oxadia-zole amine compounds $\mathbf{7 ( \mathbf { a } - \mathbf { h } )}$

Correspondence to: Adimule Vinayak

E-mail: adimulevinayak@yahoo.in

Phone: +919481268717; Fax: +9108022286386

Dhaka Univ. J. Pharm. Sci. 16(1): 11-19, 2017 (June) were obtained from compound (6) which was prepared from (5) by the in situ dehydration and cyclization reaction using phosphoryl oxychloride and chloroacetic acid under microwave irradiation. The intermediate carbohydrazide (5) was completely converted into product (6) in the form of a solid by subsequent neutralization. The starting material used for this synthetic pathway was 6-bromonicotinic acid (1) which was converted to 6-(4-fluorophenyl) nicotinic acid ethyl ester $^{8}$ (4) by treating the ethyl ester (3) with 4-fluorophenyl boronic acid in presence of tetra bis (triphenyl phosphine) palladium (0) in ethanol. The ester (4) thus obtained was converted into the corresponding hydrazide (5) by treating with hydrazine hydrate. ${ }^{9}$ The novel amine derivatives 7 (ah) obtained by refluxing compound (6) with primary or secondary amines in deoxan and TEA were purified by column chromatography (silica gel 100- 
200 mesh) and characterized by ${ }^{1} \mathrm{H}$ NMR, ${ }^{13} \mathrm{C}$ NMR and MS spectroscopic analyses. The percentage yield of all the final compounds was found to be in the range $48-88 \%$ and purity in between $94-97 \%$. The scheme of reactions from compound 1-6 is shown in figure 2. The conversion of compound (6) to amine derivatives, $7(\mathrm{a}-\mathrm{h})$ have been given in figure 3 . It was envisaged that the novel amine derivatives of 1, 3, 4oxadiazole (Figure 1, B) would enhance the solubility, total polar surface area (TPSA) and bioavailability. With this view the compounds have been subjected to MTT assay ${ }^{10}$ for testing their cytotoxic activity using 5-fluorouracil as the standard drug. Three human carcinoma cell lines were employed for the screening and the activity was calculated in terms of the $\mathrm{IC}_{50}$ values. Some of the synthesized 1,3,4-oxadiazoles were found to exhibit better cytotoxicity against $\mathrm{HepG2}$ and Caco-2cell lines.

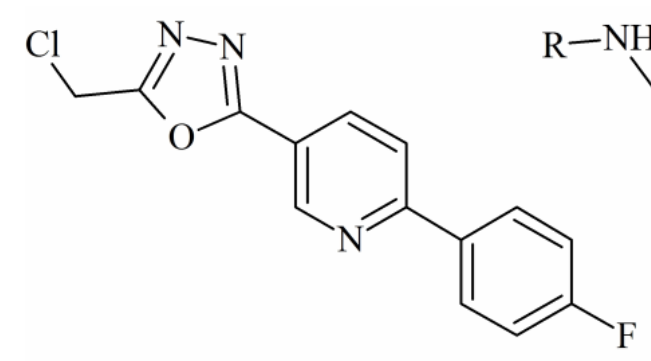

A
B

\section{$\mathrm{R}=$ Alkyl or Aryl or Anyother}

Figure 1. 5-[5-(chloromethyl)-1, 3, 4-oxadiazol-2-yl]-2-(4-fluorophenyl)-pyridine(A); Novel amine analogues of 5-[5-(chloromethyl)-1, 3, 4-oxadiazol-2-yl]-2-(4-fluorophenyl)-pyridine (B); General structure of 2,5-disubstituted 1,3,4-oxadiazole moiety (C).
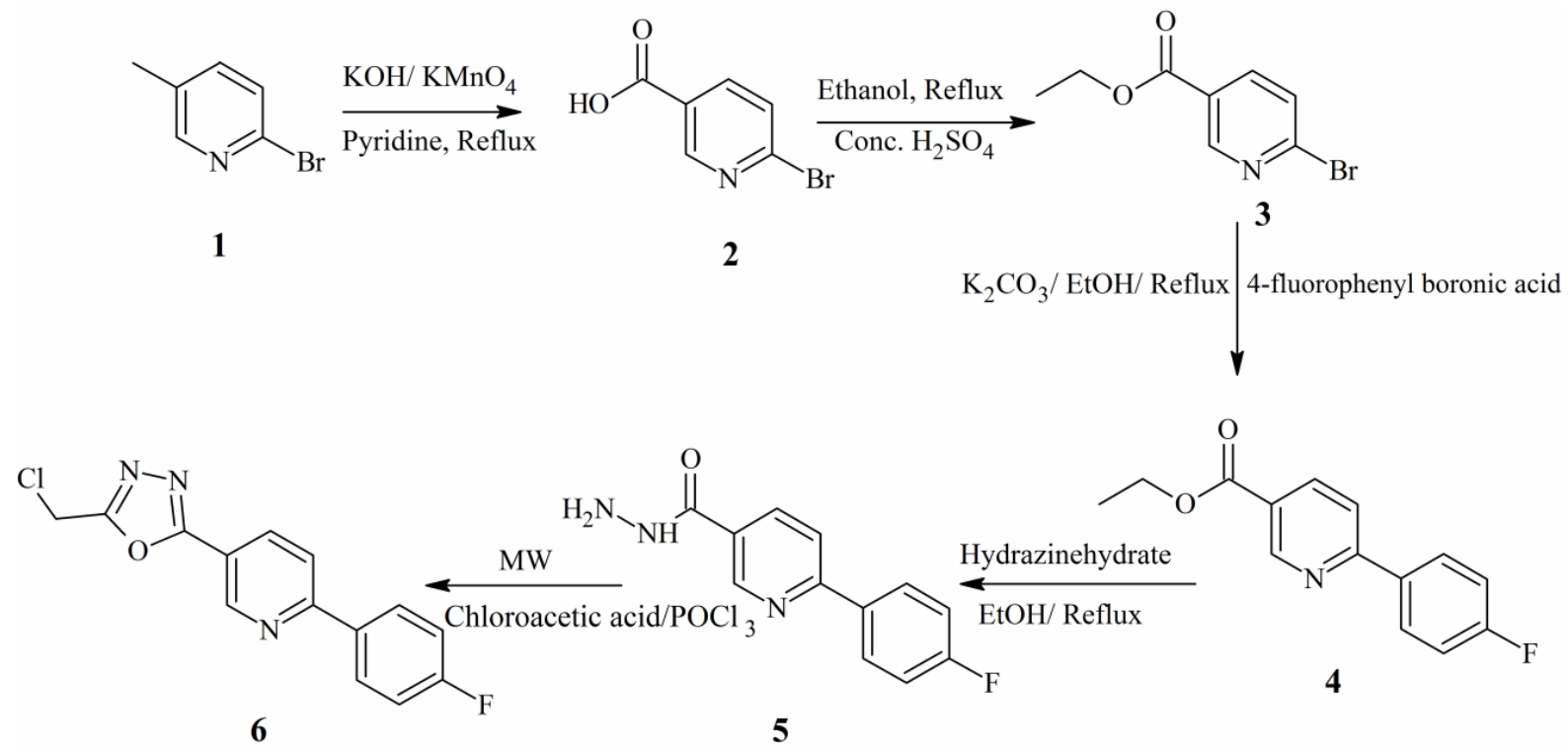

Figure 2. Scheme of synthetic pathway of the compound 5-[5-(chloromethyl)-1, 3, 4-oxadiazol-2-yl]-2-(4-fluorophenyl)-pyridine (6). 


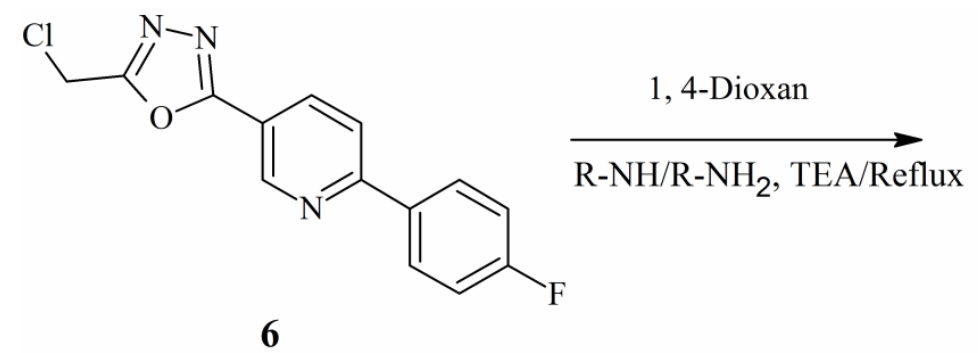

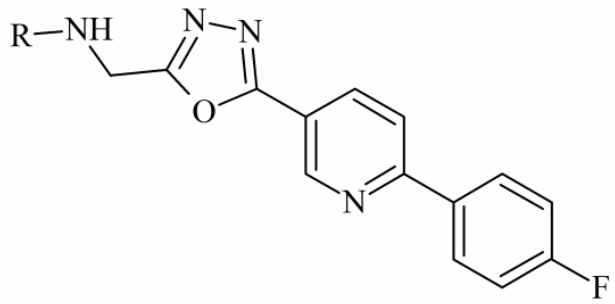

7a-h

$\mathrm{R}=$

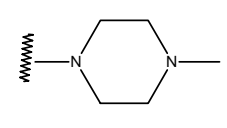

a

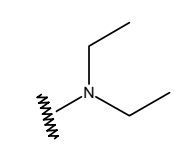

d

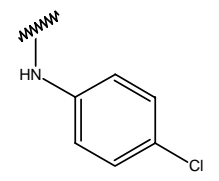

g

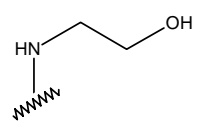

b

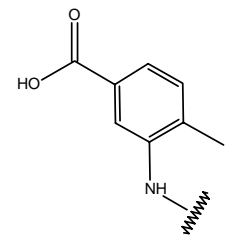

e

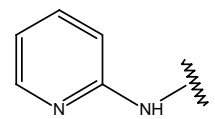

h

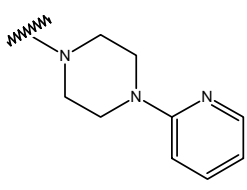

C

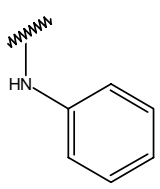

f

Figure 3. Synthetic route of the novel amine derivatives of 5-[5-(chloromethyl)-1, 3, 4-oxadiazol-2-yl]-2-(4-fluorophenyl)-pyridine 7 (a-h).

\section{MATERIALS AND METHODS}

All reagents, chemicals and solvents were purchased from S-D fine and Spectrochem Ltd., Bangalore, India. ${ }^{1} \mathrm{H}$-and ${ }^{13} \mathrm{C}$-NMR were recorded by Brucker $400 \mathrm{MHz}$ spectrophotometer. Melting points were determined using Buchi melting point apparatus 545. Mass spectra were recorded by Agilent 1200 series. TLC was done on $\mathrm{F}_{254}$ grade silica 60 from Merck. All the final and intermediate compounds were purified by normal glass column using silica gel 100-200 mesh. IR spectra were recorded by FTIR (1800S) series. Microwave reaction was carried out using Whirlpool semi-automated microwave.

Synthesis of 6-bromo-nicotinic acid (2). 2Bromo-5-methyl-pyridine (15 g, $0.0873 \mathrm{~mol}$ ) was taken in $1 \mathrm{~L} \mathrm{RB}$ flask containing $100 \mathrm{ml}$ of water and
$100 \mathrm{ml}$ of pyridine. $\mathrm{KOH}(14.65 \mathrm{~g}, 0.0261 \mathrm{~mol})$ and $\mathrm{KMnO}_{4}(68.96 \mathrm{~g}, 0.0436 \mathrm{~mol})$ were added to this reaction mixture and it was refluxed at $85^{\circ} \mathrm{C}$ overnight. After completion of the reaction as observed by TLC, solvent was removed, residue was diluted with water $(100-150 \mathrm{ml})$ and filtered. The filtrate was neutralized with $1 \mathrm{~N} \mathrm{HCl}$ and the solid that separated out was filtered, washed with water and dried. Yield $66.66 \%$; m.p. $108-112^{\circ} \mathrm{C}$; MS (ESI): $[\mathrm{M}-\mathrm{H}]^{+}$201; ${ }^{1} \mathrm{HNMR}\left(400 \mathrm{MHz}, \mathrm{CDCl}_{3}\right): \delta 7.03$ (dd,1H, $J=12.2 \mathrm{~Hz}$, Ar-H), 9.49 (dd, $J=7.2 \mathrm{~Hz}, 1 \mathrm{H})$, $9.78(\mathrm{~m}, J=15.6 \mathrm{~Hz}, 1 \mathrm{H}), 10.85$ (bs, $1 \mathrm{H}, \mathrm{OH})$.

Synthesis of 6-bromo-nicotinic acid ethyl ester (3). 6-Bromo-nicotinic acid (2) (10 g, $0.0434 \mathrm{~mol})$ was taken in $100 \mathrm{ml}$ ethanol. To this solution 10 drops of conc. $\mathrm{H}_{2} \mathrm{SO}_{4}$ were added and the mixture 
was refluxed at $80{ }^{\circ} \mathrm{C}$ for $8 \mathrm{hrs}$. TLC was used to check the completion of the reaction. After completion of the reaction, solvent was removed, residue was diluted with water and neutralised with $10 \% \mathrm{NaHCO}_{3}$ solution. The product was extracted with ethyl acetate $(15 \mathrm{ml} \times 3)$, washed with brine $(10$ $\mathrm{ml}$ ) and dried over $\mathrm{Na}_{2} \mathrm{SO}_{4}$. Ethyl acetate was concentrated to get pale yellow syrup. Yield 85\%; MS (ESI): $[\mathrm{M}+\mathrm{H}]^{+} 231 ;{ }^{1} \mathrm{HNMR}\left(400 \mathrm{MHz}, \mathrm{CDCl}_{3}\right)$ : $\delta 1.19\left(\mathrm{t}, 3 \mathrm{H}, \mathrm{CH}_{3}\right), 3.88$ (q, 2H, $\mathrm{CH}_{2}$ ), 7.14 (dd, $1 \mathrm{H}, J$ $=12.2 \mathrm{~Hz}, \mathrm{Ar}-\mathrm{H}), 9.50(\mathrm{dd}, J=8.4 \mathrm{~Hz}, 1 \mathrm{H}), 9.82(\mathrm{~m}$, $J=14.3 \mathrm{~Hz}, 1 \mathrm{H})$.

Synthesis of 6-(4-fluorophenyl)-nicotinic acid ethyl ester (4). A mixture of 6-bromonicotinic acid ethyl ester (3) (8.5 g, $0.0369 \mathrm{~mol}), \mathrm{K}_{2} \mathrm{CO}_{3}(15.27 \mathrm{~g}$, $0.1107 \mathrm{~mol})$, tetrabis triphenyl palladium (0) $(0.213$ $\mathrm{g}, 0.000185 \mathrm{~mol}$ ) and 4-fluorophenylboronic acid $(5.166 \mathrm{~g}, 0.0369 \mathrm{~mol})$ were refluxed at $85^{\circ} \mathrm{C}$ in ethanol for 3-5 hrs (Suzuki-Mayora coupling reaction). After completion of the reaction as per the TLC monitoring, solvent was removed, residue was diluted with water and the product was extracted with ethyl acetate $(25 \mathrm{ml} \times 4)$, washed with brine $(10 \mathrm{ml})$ and dried over $\mathrm{Na}_{2} \mathrm{SO}_{4}$. Ethyl acetate was concentrated to afford $6.3 \mathrm{~g}$ of crude product. The crude product was purified by column chromatography (silica gel 100-200 mesh). Purification process started with $100 \% n$-hexane and solvent polarity was increased to up $28 \%$ ethyl acetate. Yield $61.1 \%$; m.p. $128-127^{\circ} \mathrm{C}$; MS (ESI): $[\mathrm{M}+\mathrm{H}]^{+}$246; ${ }^{1} \mathrm{H}$ NMR $\left(400 \mathrm{MHz}, \mathrm{CDCl}_{3}\right): \delta 2.19$ $\left(\mathrm{t}, 3 \mathrm{H}, \mathrm{CH}_{3}\right), 3.96\left(\mathrm{q}, 2 \mathrm{H}, \mathrm{CH}_{2}\right), 7.24(\mathrm{dd}, 2 \mathrm{H}, J=$ $7.8 \mathrm{~Hz}, \mathrm{Ar}-\mathrm{H}), 8.54(\mathrm{dd}, J=8.8 \mathrm{~Hz}, 2 \mathrm{H}), 8.88$ (m, $J=$ $14.2 \mathrm{~Hz}, 3 \mathrm{H})$.

Synthesis of 6-(4-fluorophenyl)-nicotinic acid hydrazide (5). A mixture of 6-(4-fluorophenyl) nicotinic acid ethyl ester (4) (5.2 g, $0.0211 \mathrm{~mol}), 10$ $\mathrm{ml}$ of hydrazine hydrate and $50 \mathrm{ml}$ of ethanol was refluxed for overnight at $80^{\circ} \mathrm{C}$. After completion of the reaction, solvent was removed and few ice pieces and saturated brine solution were added to the residue. Solid that separated out was filtered, washed with $10 \mathrm{ml}$ of water and dried. Yield 59.6\%; m.p. 172- $178^{\circ} \mathrm{C}$; MS (ESI): $[\mathrm{M}+\mathrm{H}]^{+}{ }^{2} 232 ;{ }^{1} \mathrm{H}$ NMR (400
$\left.\mathrm{MHz}, \mathrm{CDCl}_{3}\right): \delta 2.19\left(\mathrm{t}, 3 \mathrm{H}, \mathrm{CH}_{3}\right), 3.97\left(\mathrm{q}, 2 \mathrm{H}, \mathrm{CH}_{2}\right.$ ), 4.21 (bs, $2 \mathrm{H}, \mathrm{NH}_{2}$ ), 7.32 (dd, $2 \mathrm{H}, J=11.8 \mathrm{~Hz}, \mathrm{Ar}-\mathrm{H}$ ), $8.49(\mathrm{dd}, J=8.7 \mathrm{~Hz}, 2 \mathrm{H}), 8.92(\mathrm{~m}, J=12.4 \mathrm{~Hz}, 3 \mathrm{H})$.

Synthesis of 5-[5-(chloromethyl)-1,3,4-oxadiazol-2-yl]-2-(4-fluorophenyl)-pyridine (6). A $200 \mathrm{ml}$ flask containing compound (4) (3.1 g, $1 \mathrm{mmol}$ ), phosphoryl oxychloride and chloroacetic acid was irradiated with microwave for a period of 5 minutes. TLC was monitored to check the completion. After completion of the reaction ice cold water was added to the mixture and neutralised with a saturated solution of $\mathrm{Na}_{2} \mathrm{CO}_{3}$. The separated solid was filtered, washed with $10 \mathrm{ml}$ of water and dried. The product was used without any further purification. Yield 64.5\%; MS (ESI): $[\mathrm{M}+\mathrm{H}]^{+}$; m.p. $98-104^{\circ} \mathrm{C} ;{ }^{1} \mathrm{H}$ NMR (400 MHz, $\left.\mathrm{CDCl}_{3}\right): \delta 4.56\left(\mathrm{~s}, 2 \mathrm{H}, \mathrm{CH}_{2}\right), 7.31(\mathrm{dd}, 2 \mathrm{H}$, $J=8.2 \mathrm{~Hz}, \mathrm{Ar}-\mathrm{H}), 8.54(\mathrm{dd}, J=12.2 \mathrm{~Hz}, 2 \mathrm{H}), 8.86$ (m, $J=13.2 \mathrm{~Hz}, 3 \mathrm{H}$ ).

General procedure for the synthesis of amine derivatives of 5-[5-(chloromethyl)-1,3,4-oxadiazol2-yl]-2-(4-fluorophenyl)-pyridine(7a-h). Compound (6) $(200 \mathrm{mg}, 1 \mathrm{mmol})$ was added to a mixture of 1, 4dioxin $(10 \mathrm{ml})$, TEA $(2 \mathrm{ml})$ and the corresponding amines (a-h, $1.1 \mathrm{mmol}$ ) refluxed for 3-8 hrs. TLC was used to check the completion of the reaction. After completion, the solvent was removed, residue was diluted with water $(10 \mathrm{ml})$ and the product was extracted with ethyl acetate $(10 \mathrm{ml} \times 2)$, washed with brine $(10 \mathrm{ml})$ and dried over anhydrous $\mathrm{Na}_{2} \mathrm{SO}_{4}$. The crude product was purified by column chromatography (silica gel 100-200 mesh) using ethyl acetate-hexane $(50: 50)$.

Analytical data of the novel amine derivatives of 5-[5-(chloromethyl)-1,3,4-oxadiazol-2-yl]-2-(4fluorophenyl)-pyridine (7a-h). 1-\{5-[6-(4-Fluorophenyl)-pyridin-3-yl]-[1,3,4] oxadiazol-2-yl-methyl $\}$ 4-methyl-piperazine 7(a).

Brown syrup; yield 68\%; ${ }^{1} \mathrm{H}$ NMR (400 MHz, $\mathrm{CDCl}_{3}$ ): $\delta 2.27\left(\mathrm{~s}, 3 \mathrm{H}, \mathrm{CH}_{3}\right), 2.46\left(\mathrm{bs}, 4 \mathrm{H}, \mathrm{CH}_{2}\right), 2.48$ (bs, $\left.4 \mathrm{H}, \mathrm{CH}_{2}\right), 3.2\left(\mathrm{~s}, 2 \mathrm{H}, \mathrm{CH}_{2}\right), 7.2(\mathrm{dd}, 2 \mathrm{H}, J=7.4$ $\mathrm{Hz}), 7.34(\mathrm{dd}, 2 \mathrm{H}, J=11.4 \mathrm{~Hz}, \mathrm{ArH}), 7.58(\mathrm{~s}, 1 \mathrm{H}$, ArH), $7.71(\mathrm{~m}, 2 \mathrm{H}, \quad J=14.8 \mathrm{~Hz}) ;{ }^{13} \mathrm{C}$ NMR $(100$ $\left.\mathrm{MHz}, \mathrm{CDCl}_{3}\right)$ : 38.7, 53.6, 55.1, 58, 116.0, 122.6, $124.8,128.7,130.3,135.3,142.2,155.7,161.7$; IR 
$\left(\mathrm{KBr}, v_{\max } / \mathrm{cm}^{-1}\right): 2905,3326,1108,894,2715,3311$; MS (ESI): $[\mathrm{M}+\mathrm{H}]^{+}$354, anal. calculated for $\mathrm{C}_{19} \mathrm{H}_{20} \mathrm{FN}_{5} \mathrm{O} ; \mathrm{C}, 64.57 ; \mathrm{H}, 5.70 ; \mathrm{F}, 5.38 ; \mathrm{N}, 19.82 ; \mathrm{O}$, 4.57; Found C, 64.59; H, 5.73; F, 5.39; N, 19.83; O, 4.61 .

2-(\{5-[6-(4-Fluorophenyl)-pyridin-3-yl]-[1,3,4] oxadiazol-2-yl-methyl\}-amino)-ethanol (7b). Pale brown syrup; yield 53\%; ${ }^{1} \mathrm{H}$ NMR (400 $\mathrm{MHz}$, $\left.\mathrm{CDCl}_{3}\right): \delta 2.74\left(\mathrm{~s}, 2 \mathrm{H}, \mathrm{CH}_{2}\right), 3.65\left(\mathrm{~s}, 2 \mathrm{H}, \mathrm{CH}_{2}\right), 3.81$ (s, 2H, $\left.\mathrm{CH}_{2}\right), 4.2$ (bs, 1H, OH), 7.47 (dd, 2H, $J=7.2$ $\mathrm{Hz}$, Ar-H), 7.54 (dd, $J=8.9 \mathrm{~Hz}, 2 \mathrm{H}), 7.64(\mathrm{~m}, J=$ $13.3 \mathrm{~Hz}, 2 \mathrm{H}), 8.14(\mathrm{~s}, 1 \mathrm{H}), 10.8(\mathrm{~s}, 1 \mathrm{H}, \mathrm{NH}) ;{ }^{13} \mathrm{C}$ NMR $\left(100 \mathrm{MHz}, \mathrm{CDCl}_{3}\right): \delta 49.5,52,64.9,116$, $120.8,122.3,124.8,128.7,131.1,135.3,142.3$, 148.8, 149.6, 152.3, 160.7; IR $\left(\mathrm{KBr} v_{\max } / \mathrm{cm}^{-1}\right): 2935$, 3236, 1210, 873, 2885, 3420; MS (ESI) : [M-H] $]^{+} 312$; anal. calculated for $\mathrm{C}_{16} \mathrm{H}_{15} \mathrm{FN}_{4} \mathrm{O}_{2} ; \mathrm{C}, 61.14 ; \mathrm{H}, 4.81$; F, 6.04; N, 17.83; O, 10. 19; found C, 61.16; H, 4.84; F, 6.06; N, 17.85; O, 10.29 .

1-\{5-[6-(4-Fluorophenyl)-pyridin-3-yl]-[1,3,4] oxadiazol-2-yl-methyl\}-4-pyridin-2-yl-piperazine (7c). Off white solid; yield $52 \%$; m. p. $203-204^{0} \mathrm{C} ;{ }^{1} \mathrm{H}$ NMR (400 MHz, $\mathrm{CDCl}_{3}$ ): $\delta 2.59$ (bs, $4 \mathrm{H}, \mathrm{CH}_{2}$ ), 3.16 (bs, 4H, $\left.\mathrm{CH}_{2}\right), 3.62$ (s, 2H, $\left.\mathrm{CH}_{2}\right), 7.13$ (dd, $J=4.9$ $\mathrm{Hz}, 2 \mathrm{H}, \mathrm{ArH}), 7.32$ (dd, $J=7.2 \mathrm{~Hz}, 2 \mathrm{H}), 7.54$ (m, $J=$ $12.8 \mathrm{~Hz}, 2 \mathrm{H}), 7.81(\mathrm{~m}, J=14.2 \mathrm{~Hz}, 2 \mathrm{H}), 7.84$ (dd, $J=$ $8.4 \mathrm{~Hz}, 2 \mathrm{H}), 8.10(\mathrm{~s}, 1 \mathrm{H}), 9.35(\mathrm{~s}, 1 \mathrm{H}, \mathrm{NH}) ;{ }^{13} \mathrm{C} \mathrm{NMR}$ $\left(100 \mathrm{MHz}, \mathrm{CDCl}_{3}\right): \delta 48.7,53.2,55,57.9,61.1$, $108.8,112,113.9,116.3,122.7,125.5,128.7,132.2$, $135.4,138.8,142.3,148.9,153.2,155.8,161.9$, 168.9; $\operatorname{IR}\left(\mathrm{KBr}, v_{\max } / \mathrm{cm}^{-1}\right): 2913,3326,2865,3380$; MS (ESI): $[\mathrm{M}+\mathrm{H}]^{+}$418; anal. calculated for $\mathrm{C}_{23} \mathrm{H}_{21} \mathrm{FN}_{6} \mathrm{O} ; \mathrm{C}, 66.33 ; \mathrm{H}, 5.08 ; \mathrm{F}, 4.56 ; \mathrm{N}, 20.18$; O, 3.84; found $\mathrm{C}, 66.35 ; \mathrm{H}, 5.09 ; \mathrm{F}, 4.58 ; \mathrm{N}, 20.19$; O, 3.85 .

Diethyl-\{5-[6-(4-fluorophenyl)-pyridin-3-yl][1,3,4] oxadiazol-2-yl-methyl\}-amine (7d). Pale semisolid; yield $71 \%$; ${ }^{1} \mathrm{H}$ NMR (400 MHz, $\mathrm{CDCl}_{3}$ ): $\delta$ $1.1\left(\mathrm{~s}, 6 \mathrm{H}, 2 \mathrm{CH}_{3}\right), 2.40\left(\mathrm{~s}, 4 \mathrm{H}, \mathrm{CH}_{2}\right), 3.62(\mathrm{~s}, 2 \mathrm{H}$, $\left.\mathrm{CH}_{2}\right), 7.32(\mathrm{dd}, J=14.2 \mathrm{~Hz}, 2 \mathrm{H}), 7.48(\mathrm{dd}, J=7.8$ $\mathrm{Hz}, 2 \mathrm{H}), 7.54$ (m, $J=14.8 \mathrm{~Hz}, 1 \mathrm{H}, \mathrm{Ar}-\mathrm{H}), 7.75$ (dd, $J$ $=8.8 \mathrm{~Hz}, 2 \mathrm{H}), 9.81(\mathrm{~s}, 1 \mathrm{H}, \mathrm{NH}) ;{ }^{13} \mathrm{C}$ NMR $(100$ $\left.\mathrm{MHz}, \mathrm{CDCl}_{3}\right): \delta 13.7,24.5,46.3,53.7,116.7,118.8$, 122.2, 128.6, 130.5, 135.4, 142.3, 149.3, 151.8,158.8,
162.9; IR (KBr, $\left.v_{\max } / \mathrm{cm}^{-1}\right): 2983,3486,1250,881$, 2795, 3311; MS (ESI): $[\mathrm{M}+\mathrm{H}]^{+} 327$; anal. calculated for $\mathrm{C}_{18} \mathrm{H}_{19} \mathrm{FN}_{4} \mathrm{O} ; \mathrm{C}, 66.24 ; \mathrm{H}, 5.87 ; \mathrm{F}, 5.82 ; \mathrm{N}, 17.17$; $\mathrm{O}, 4.90$; found $\mathrm{C}, 66.25 ; \mathrm{H}, 5.89 ; \mathrm{F}, 5.84 ; \mathrm{N}, 17.18$; O, 4.98 .

3-(\{5-[6-(4-Fluorophenyl) pyridin-3-yl]-[1,3,4] oxadiazol-2-yl-methyl\}-amino)-4-methyl- benzoic acid (7e). White solid; yield 48\%;m.p. $143-148^{0} \mathrm{C} ;{ }^{1} \mathrm{H}$ NMR (400 MHz, $\left.\mathrm{CDCl}_{3}\right): \delta 2.35\left(\mathrm{~s}, 3 \mathrm{H}, \mathrm{CH}_{3}\right), 4.32$ (s, $\left.2 \mathrm{H}, \mathrm{CH}_{2}\right), 4.8(\mathrm{~s}, 1 \mathrm{H}, \mathrm{NH}), 7.33 \mathrm{dd}, J=5.2 \mathrm{~Hz}$, 2H), 7.41 (dd, $J=7.3 \mathrm{~Hz}, 2 \mathrm{H}), 7.49(\mathrm{~m}, J=14.2 \mathrm{~Hz}$, 2H, Ar-H), 7.65 (dd, $J=4.2 \mathrm{~Hz}, 1 \mathrm{H}), 8.18$ (m, $J=$ $14.2 \mathrm{~Hz}, 3 \mathrm{H}), 10.8$ (bs, $1 \mathrm{H}, \mathrm{OH}) ;{ }^{13} \mathrm{C}$ NMR $(100$ $\left.\mathrm{MHz} \mathrm{CDCl}_{3}\right)$ : 12.1, 51.9, 116.2, 118.9, 119.3, 122.3, $122.6,127.8,128.7,133.4,135.3,140.5,144.1$, 149.6, 152.3, 160.7, 162.3, 169.8, 170.8, 174.2; IR $\left(\mathrm{KBr}, v_{\max } / \mathrm{cm}^{-1}\right): 2865,3476,1288,849,2795,3279$; MS (ESI): $[\mathrm{M}-\mathrm{H}]^{+}$403; anal.calculated for $\mathrm{C}_{22} \mathrm{H}_{17} \mathrm{FN}_{4} \mathrm{O}_{3} ; \mathrm{C}, 65.34 ; \mathrm{H}, 4.24 ; \mathrm{F}, 4.70 ; \mathrm{N}, 13.85$; $\mathrm{O}, 11.87$; found $\mathrm{C}, 65.36 ; \mathrm{H}, 4.25 ; \mathrm{F}, 4.72 ; \mathrm{N}, 13.87$; $\mathrm{O}, 11.89$.

\{5-[6-(4-Fluorophenyl)-pyridin-3-yl]-[1,3,4] oxadiazol-2-yl-methyl\}-phenyl-amine(7f). Brown coloured solid; yield $38 \%$; m. p. $96-102{ }^{\circ} \mathrm{C}$; ${ }^{1} \mathrm{HNMR}$ $\left(400 \mathrm{MHz}, \mathrm{CDCl}_{3}\right): \delta 4.32$ (s, $\left.2 \mathrm{H}, \mathrm{CH}_{2}\right), 4.78$ (bs, $1 \mathrm{H}$, $\mathrm{NH}), 7.28(\mathrm{dd}, J=14.1 \mathrm{~Hz}, 2 \mathrm{H}), 7.36(\mathrm{dd}, J=8.7 \mathrm{~Hz}$, 2H), $7.48(\mathrm{~m}, J=13.2 \mathrm{~Hz}, 3 \mathrm{H}, \mathrm{Ar}-\mathrm{H}), 7.65$ (dd, $J=$ $7.8 \mathrm{~Hz}, 2 \mathrm{H}), 7.76(\mathrm{~m}, J=12.2 \mathrm{~Hz}, 3 \mathrm{H}) ;{ }^{13} \mathrm{C}$ NMR $\left(100 \mathrm{MHz}, \mathrm{CDCl}_{3}\right): \delta$ 51.6, 112.3, 114.5, 116.7, $120.6,122.4,129.3,131.7,135.8,142.2,149.6$, 155.7, 160.8, 164.5; IR $\left(\mathrm{KBr}, v_{\max } / \mathrm{cm}^{-1}\right): 2815,3426$, 1360, 889, 2755, 3359; MS (ESI) $[\mathrm{M}+\mathrm{H}]^{+} 347$; anal. calculated for $\mathrm{C}_{20} \mathrm{H}_{15} \mathrm{FN}_{4} \mathrm{O} ; \mathrm{C}, 69.35 ; \mathrm{H}, 4.37 ; \mathrm{F}$, 5.49; N, 16.18; O, 4.62; Found C, 69.37; H, 4.39; F, $5.51 ; \mathrm{N}, 16.19 ; \mathrm{O}, 4.64$.

(4-Chlorophenyl)-\{5-[6-(4-fluorophenyl)-

pyridin-3-yl]-[1,3,4] oxadiazol-2-yl-methyl\}-amine (7g). White coloured solid; yield 64\%; m. p. 128$129^{\circ} \mathrm{C} ;{ }^{1} \mathrm{HNMR}\left(400 \mathrm{MHz}, \mathrm{CDCl}_{3}\right): \delta 7.31(\mathrm{dd}, J=$ $7.2 \mathrm{~Hz}, 2 \mathrm{H}), 7.43(\mathrm{dd}, J=12.5 \mathrm{~Hz}, 2 \mathrm{H}), 7.48(\mathrm{~m}, J=$ $14.2 \mathrm{~Hz}, 2 \mathrm{H}, \mathrm{Ar}-\mathrm{H}), 7.65(\mathrm{~m}, J=14.3 \mathrm{~Hz}, 2 \mathrm{H}), 7.88$ $(\mathrm{dd}, J=8.8 \mathrm{~Hz}, 2 \mathrm{H}), 8.35(\mathrm{~s}, 1 \mathrm{H}), 9.5(\mathrm{~s}, 1 \mathrm{H}, \mathrm{NH})$; ${ }^{13} \mathrm{C}$ NMR $\left(100 \mathrm{MHz}, \mathrm{CDCl}_{3}\right): \delta 51.6,112.0,113.7$, $122.2,124.5,128.6,130.3,134.5,142.2,149.6$, 
155.7, 161.8, 162.7; IR (KBr, $\left.v_{\max } / \mathrm{cm}^{-1}\right): 2834,3446$, 1480, 879, 2795, 3329; MS (ESI): [M+H] $]^{+} 382$; anal. calculated for $\mathrm{C}_{20} \mathrm{H}_{14} \mathrm{ClFN}_{4} \mathrm{O} ; \mathrm{C}, 63.08 ; \mathrm{H}, 3.71 ; \mathrm{Cl}$, 9.31; F, 4.99; N, 14.71; O, 4.20; Found C, 63.09; H, 3.74; Cl, 9.33; F, 5.01; N, 14.72; O, 4.22.

$\{5-[6-(4-F l u o r o p h e n y l)$-pyridin-3-yl]-[1,3,4] oxadiazol-2-yl-methyl $\}$-pyridin-2-yl-amine $(\mathbf{7 h})$. Pale yellow coloured solid; yield $48 \%$; m. p. $132-136^{\circ} \mathrm{C}$; ${ }^{1} \mathrm{H}$ NMR (400 MHz, $\left.\mathrm{CDCl}_{3}\right): \delta 7.31(\mathrm{dd}, J=7.2 \mathrm{~Hz}$, 2H), 7.43 (dd, $J=14.2 \mathrm{~Hz}, 2 \mathrm{H}), 7.48$ (m, $J=12.8 \mathrm{~Hz}$, 3H, Ar-H), 7.65 (m, $J=12.8 \mathrm{~Hz}, 2 \mathrm{H}), 7.88$ (dd, $J=$ $5.2 \mathrm{~Hz}, 1 \mathrm{H}), 9.5(\mathrm{~s}, 1 \mathrm{H}, \mathrm{NH}) ;{ }^{13} \mathrm{C}$ NMR $\left(\mathrm{CDCl}_{3}, 100\right.$ $\mathrm{MHz}): \delta$ 51.6, 52.2, 108.3, 112.0, 113.7, 120.8, $122.6,130.8,132.2,141.2,144.5,149.6,160.7$, 164.3; IR (KBr, $\left.v_{\max } / \mathrm{cm}^{1}\right): 2855,3486,1480,884$, 2795, 3379; MS (ESI): $[\mathrm{M}+\mathrm{H}]^{+} 347$; anal. calculated for $\mathrm{C}_{19} \mathrm{H}_{14} \mathrm{FN}_{5} \mathrm{O} ; \mathrm{C}, 65.70 ; \mathrm{H}, 4.06 ; \mathrm{F}, 5.47 ; \mathrm{N}, 20.16$; O, 4.61; Found C, 65.72; H, 4.08; F, 5.49; N, 20.18; $\mathrm{O}, 4.63$.

\section{Cytotoxic evaluation}

MTT assay. Cytotoxicity of the novel amine derivatives of 1, 3, 4-oxadiazoles has been determined using 3-(4, 5-dimethylthiazol-2-yl)-2, 5diphenyltetrazolium bromide (MTT) assay which was carried out at Genelon Institute of Life Sciences Pvt. Ltd. The in vitro anti-proliferative MTT assay was performed against three human carcinoma cell lines namely, HeLa, Caco-2 and HepG2. All the cell lines were grown in DMEM-HG supplemented with $10 \%$ heat-inactivated FBS, $2 \%$ penicillin-streptomycin and $2.5 \mu \mathrm{g} / \mathrm{ml}$ amphotericin-B solutions (all from $\mathrm{HI}$ Media Labs, Mumbai, India). Cell lines were incubated at $37^{\circ} \mathrm{C}$ in a humidified atmosphere with $95 \%$ air, $5 \% \mathrm{CO}_{2}$. Following 24-48 hrs of incubation period, the adherent cells were detached using Trypsin-EDTA solution. Cell count was done using the Luna automated cell counter (Logos Bio systems, India) based on trypan blue dye exclusion method.

Cell viability assay. The MTT assay was carried out using the following procedure. Cell suspension $(200 \mu \mathrm{l})$ was seeded in 96-well micro plates (Corning ${ }^{\circledR}$, USA) at a density of 25,000 cells/well and incubated for $24 \mathrm{hrs}$. All cells were seeded in duplicates with novel compounds $\mathbf{7 a - 7 h}$ having range of concentrations from $50-500 \mu \mathrm{M}$, incubated in a $\mathrm{CO}_{2}$ incubator at $37^{\circ} \mathrm{C}$. Treated cells were there after incubated with $10 \%$ MTT $(5 \mathrm{mg} / \mathrm{ml}$; HI Media Labs, Mumbai, India) for $3 \mathrm{hrs}$. The culture medium was then aspirated and $200 \mu$ l dimethyl sulfoxide (DMSO; Sigma-Aldrich, India) was added to it. 5-Fluorouracil (5-FU) was used as the standard. Cell viability was determined by measuring the absorbance on a micro plate reader (BMG Labtech, Germany) at $570 \mathrm{~nm}$ and calculated as a percentage of viable cells at different test concentrations relative to the control (5-FU).

$\left[\%\right.$ cell viability $=\left(\mathrm{A}_{570}\right.$ of treated cells $/ \mathrm{A}_{570}$ of control cells) $\times 100 \%]$.

\section{RESULTS AND DISCUSSION}

A series of novel derivatives of 2-(4fluorophenyl)-5-(5-aryl substituted-1,3,4-oxadiazol2-yl) pyridine (7a-h) were synthesized, characterized and evaluated for their cytotoxic effect ${ }^{11}$ against HeLa, Caco-2 and HepG2 cell lines. The synthesis started with 2-bromo-5-methyl-pyridine which was converted into 6-bromonicotinic acid (2) ${ }^{12,13}$ (the shifting of the CO in IR was $1180 \mathrm{~cm}^{-1}$ ). Compound 2 was converted into corresponding ethyl ester which was further reacted with 4-fluorophenyl boronic acid by Suzuki-Mayora coupling reaction. Compound 4 thus obtained was converted into corresponding carbohydrazide by refluxing with hydrazine hydrate and ethanol (IR absorbance of NH at $3395 v_{\max } / \mathrm{cm}^{-1}$ ) and appearance of broad $\mathrm{NH}_{2}$ peak at $\left.\delta 4.23\right)$. The intermediate 6-(4-fluorophenyl) nicotinic acid hydrazide were reacted with phosphorous oxychloride in presence of monochloroacetic acid to yield cyclised product (6). The intermediate 6 was reacted with various primary and secondary amines to afford the final compounds (7a-h). The compounds were screened against three cancer cell lines (MTT assay). The in vitro anticanceractivity ${ }^{14,15}$ of these compounds was expressed in the form of inhibitory concentration $\left(\mathrm{IC}_{50}\right)$. The different substituted 1,3,4-oxadiazoles derivatives of pyridine ${ }^{16,17}$ moiety showed broad range of viability against $\mathrm{CaCo}-2$ and $M C F 7$ cell lines. 
Table 1. IC $_{50}$ values of the synthesized novel amine derivatives of 5-[5- (chloromethyl)-1, 3, 4-oxadiazol-2-yl]-2-(4-fluorophenyl)pyridine (7a-h).

\begin{tabular}{lccc}
\hline Compound No & $\mathrm{IC}_{50}{ }^{\#}$ values of $7(\mathrm{a}-\mathrm{h})$ in $(\mu \mathrm{M})$ \\
\hline 7a-h & $H e L a$ & Caco-2 & HepG2 \\
\hline 7a & $212.4 \pm 1.2$ & $203.6 \pm 2.3$ & $2.6 \pm 0.5$ \\
7b & $85.6 \pm 0.8$ & $112.5 \pm 1.2$ & $45.6 \pm 1.1$ \\
7c & $34.8 \pm 1.3$ & $123.8 \pm 1.4$ & $128.9 \pm 3.5$ \\
7d & $112.9 \pm 0.4$ & $145.6 \pm 0.4$ & $5.8 \pm 1.6$ \\
7e & $118.4 \pm 0.5$ & $212.3 \pm 0.4$ & $32.2 \pm 0.3$ \\
7f & $78.3 \pm 5.4$ & $2.3 \pm 0.5$ & $23.5 \pm 4.6$ \\
7g & $56.4 \pm 3.4$ & $56.8 \pm 1.2$ & $156.7 \pm 2.3$ \\
7h & $88.6 \pm 1.2$ & $34.6 \pm 0.9$ & $176.4 \pm 1.6$ \\
5-FU & $7.6 \pm 0.3$ & $8.8 \pm 0.6$ & $7.6 \pm 0.2$ \\
\hline
\end{tabular}

\# Inhibitory concentration at $50 \%$ of the viable cells

\pm Average value of the two independent experiments

Table 2. $\mathrm{CC}_{50}$ of novel amine derivatives of 5-[5- (chloromethyl)-1,3,4-o xadiazol-2-yl]-2-(4-fluorophenyl)-pyridine (7a-h).

\begin{tabular}{lccc}
\hline Compound no. & \multicolumn{3}{c}{$\mathrm{CC}_{50}{ }^{*}$ of the compounds 7(a-h) } \\
\cline { 2 - 4 } 7a-h & HeLa & Caco-2 & HepG2 \\
\hline 7a & $120 \pm 1.2$ & $112 \pm 1.3$ & $34 \pm 0.5$ \\
$7 \mathrm{~b}$ & $76 \pm 0.6$ & $145 \pm 1.1$ & $129 \pm 0.3$ \\
$7 \mathrm{c}$ & 200 & $178 \pm 2.3$ & $102 \pm 1.1$ \\
$7 \mathrm{~d}$ & 450 & $100 \pm 2.6$ & $112 \pm 1.4$ \\
$7 \mathrm{e}$ & $56 \pm 2.4$ & $62 \pm 1.2$ & $76 \pm 3.4$ \\
$7 \mathrm{f}$ & $127 \pm 3.4$ & $87 \pm 2.6$ & $77 \pm 0.4$ \\
$7 \mathrm{~g}$ & 200 & $23 \pm 1.5$ & $91 \pm 4.3$ \\
7h & $123 \pm 2.3$ & $156 \pm 0.4$ & $73 \pm 1.1$ \\
5-FU & $57 \pm 0.3$ & $69 \pm 2.3$ & $52 \pm 1.8$ \\
\hline
\end{tabular}

Concentration of compound at $50 \%$ of the remaining viable cells.

Table 3. Selectivity index (SI) of the novel amine derivatives.(7a-h).

\begin{tabular}{lccc}
\hline Compound no. & \multicolumn{2}{c}{ SI of the novel amine derivatives 7 (a-h) } \\
\hline 7a-h & HeLa & Caco-2 & HepG2 \\
\hline 7a & 0.566 & 0.551 & 13.06 \\
$7 \mathrm{~b}$ & 0.887 & 1.288 & 2.828 \\
$7 \mathrm{c}$ & 5.747 & 1.437 & 0.791 \\
$7 \mathrm{~d}$ & 3.985 & 0.686 & 19.31 \\
$7 \mathrm{e}$ & 0.472 & 0.292 & 0.236 \\
$7 \mathrm{f}$ & 1.621 & 37.8 & 3.276 \\
$7 \mathrm{~g}$ & 3.546 & 0.404 & 0.580 \\
$7 \mathrm{~h}$ & 1.388 & 4.508 & 0.413 \\
5-FU & 7.5 & 7.84 & 6.84 \\
\hline
\end{tabular}


Compounds 7a and $\mathbf{7 d}$ showed very good cytotoxicity $^{18-20}$ with selectivity index (SI) against Hep $G 2$ cell lines having $\mathrm{IC}_{50}$ of $2.6 \mu \mathrm{M}$ (SI-13.06) and $5.8 \mu \mathrm{M}$ (SI-19.31), respectively. The compound 7f (SI-37.8) showed cytotoxicity against $\mathrm{Caco}-2$ cell line having $\mathrm{IC}_{50}$ of $2.3 \mu \mathrm{M}$. Overall the synthesized 1 , 3,4-oxadiazoles derivatives showed better cytotoxicity against HepG2 and Caco-2 cell lines.

\section{CONCLUSION}

In the present study novel amine derivatives of 2-(4-fluorophenyl)-5-(5-aryl substituted-1,3,4oxadiazol-2-yl) pyridine have been synthesised. These compounds have showed good cytotoxicity against HepG2 and Caco-2 cell lines. The $\mathrm{IC}_{50}$ values of the compounds7aand $7 \mathbf{d}$ against $H e p G 2$ was found to be $2.6 \mu \mathrm{M}$ (SI 13.06) and $5.8 \mu \mathrm{M}$ (SI 19.31), respectively. On Caco-2 cell lines the compound $7 f$ exhibited good cytotoxicity having $\mathrm{IC}_{50} 2.3 \mu \mathrm{M}$ and $\mathrm{CC}_{50} 87 \mu \mathrm{M}$ (SI 37.8).

\section{ACKNOWLEDGEMENTS}

The first author is thankful to Mount Carmel College (Autonomous) for the support given for this research work. We are also thankful to IPC, IISc, Bengaluru, India for providing the analytical data.

\section{REFERENCES}

1. Nagalakshmi, G. 2008. Synthesis, antimicrobial and antiinflammatory activity of 2,5-disubstituted-1,3,4-oxadiazoles. Indian. J. Pharm. Sci. 70, 49-55.

2. Jignesh, P.R., Tarunkumar, N.A., Dhaval, M.J., Kruti, N.M and Nilesh, H.P. 2014. Synthesis and in vitro antibacterial activity of new oxoethylthio-1,3,4-oxadiazole derivatives. $J$. Saudi. Chem. Soc. 18, 101-106.

3. Azimvand, J. 2012. Synthesis of new triazole and oxadiazole containing compound in the azide reaction as an antibacterial drug. J. Chem. Pharm. Res. 4, 3900-3904.

4. Janin, Y., Janin. 2007. Antituberculosis drugs ten years of research. Bioorg. Med. Chem. 15, 2479-2513.

5. Aboraia, A. S., Abdel-Rahman, H. M., Mahfouz, N.M and El-Gendy, M.A. 2006. Novel 5-(2-hydroxyphenyl)-3substituted-2,3-dihydro-1,3,4-oxadiazole-2-thione derivatives promising anticancer agents. Bioorg. Med. Chem. 14, 12361246.
6. Suzuki, N.M., Tamotsu, A., Shunzo, K., Hideyuki, T., Hideo, T., Masao, R., Yuichi, T. and Wataruisoda, S. 1992. Synthesis and allergy activity of $[1,3,4]$ thiadiazole [3,2-a]1,2,3-triazolo [4,5-d]-pyrimidin-9 (3H)-one derivatives. Chem. Bull. Res. 40, 357-63.

7. Palizban, A.A., Sadeghi-aliabadi, H. and Abdollahpour, F.2010. Effect of cerium lanthanide on $\mathrm{HeLa}$ and MCF-7 cancer cell growth in the presence of transferring. Res. Pharm. Sci. 5, 119-125.

8. Robert, G.H., Joan, H.H., Xiao-Fa Lin, Counde, O.Y., Lubica, R., Teresa Alejandra, T. M. and Less 2011 Jun 23, WO 2011073269A1.

9. Tanisha, D.B., Bruce, C.H., Wei Huang, James, R.K., Joseph, B.M., Bradley, E.N., Kirk, L.O., Matthew, J., Barbara, A.S.,Alli, T., John, I.T. and Steven, R.T.19 2008. US 20080146569A1.

10. Gursoy, A. and Karal, N. 2003. Synthesis and primary cytotoxicity evaluation of 3-[(3-phenyl-4(3H)-quinazolinone2-yl)-mercaptoacetyl]hydrazono]-1H-2-indolinones. Eur. J. Med. Chem. 38, 633-643.

11. Mosmann, T., 1983. Rapid colorimetric assay for cellular growth and survival: application to proliferation and cytotoxicity assays. J. Immunol. Methods. 65, 55-63.

12. Suzuki, N.M., Tamotsu, A., Shunzo, Eur. Pat. Appl. 1985. EP 159707 A2 19851030.

13. Rakesh, K.M., Shaharyar, A.K., and Raiand, S.C. 2013. Synthesis and biological evaluation of some novel 1,3,4oxadiazoles derived from biphenyl 4- carboxylic acid. Der. Pharmacia Lettre. 5, 366-370.

14. Karal, N., Gursoy, A., Kandemirli, F., Shvets, N., Kaynak, F.B., Ozbey, S., Kovalishyne, V. and Dimoglo, A. 2007. Synthesis and structure-antituberculosis activity relationship of 1H-indole-2, 3-dione derivatives. Bioorg. Med. Chem. 15, 5888-5904.

15. Monks, A., Scudiero, D., Skehan, P., Shoemaker, R., Paull, K., Vistica, D., Hose, C., Langley, J. and Cronise, P. 1991. Feasibility of a high-flux anticancer drug screen using a diverse panel of cultured human tumour cell lines. J. Natl. Cancer. Inst. 83, 757-766.

16. Abdel, K.M., Mohga, M.E and Nasser, S.A.M.K. 2003. Synthesis and reactions of some new heterocyclic carbohydrazides and related compounds as potential anticancer agents, Molecules 8, 744-755.

17. Skehan, P., Storeng, R., Scudiero, D., Monks, A., McMohan J.,Vistica, D., Warren, J. T., Bokesch H., Kenney, S. and Boyd, M.R. 1990. New colorimetric cytotoxicity assay for anticancer drug screening. J. Natl. Cancer Inst. 82, 11071112. 
18. Pooja, M., Suroor, A.K., Surajpal, V and Ozair. A. 2011. Thiadiazole derivatives as potential anticonvulsant agents. Bull. Korean. Chem. Soc. 32, 1011-1016.

19. Rajyalakshmi, G. Rama, N.R. Anreddy and Sarangapani, M. 2011. Synthesis, characterization and anticancer activity of certain 3-\{4-(5-mercapto-1,3,4-oxadiazole-2-yl) phenylimino\} indolin-2-one derivatives. Saudi. Pharm. J. 19, 153158.
20. Leite, L.F., Ramos, M.N., da Silva, J.B., Miranda, A.L., Fraga, C.A. and Barreiro, E.J.1999. Synthesis and analgesic profile of novel N-containing heterocycles derivatives: arylidene 3-phenyl-1,2,4-oxadiazole-5-carbohydrazide. Farmaco. 54, 747-57. 